\title{
Computationally efficient energy management of a planetary gear hybrid electric vehicle
}

\author{
Nikolce Murgovski, Xiaosong Hu, Bo Egardt \\ Department of Signals and Systems, Chalmers University of \\ Technology, 41296 Gothenburg, Sweden (e-mail: \\ nikolce.murgovski@chalmers.se).
}

\begin{abstract}
We present a method for obtaining a computationally efficient, sub-optimal energy management of an electrified vehicle containing a planetary gear set. We first reformulate the optimization problem to become separable in space (optimization variables). The problem is then decomposed into two optimization problems. The first is a static problem that looks for the optimal engine speed that maximizes efficiency of a compound unit, resembling an enginegenerator unit combining the planetary gear and kinetic energy converters connected to it. The second is a dynamic optimization problem deciding the optimal power split between an electric buffer and the compound unit. By approximating the losses of the compound unit as convex, second order polynomial in generated power, we are able to solve the power split problem in less than 2 seconds, when the engine on/off sequence is known in advance. By comparing results with dynamic programming, we observed an approximation error of less than $0.2 \%$.
\end{abstract}

Keywords: electrified vehicle, convex optimization, energy management, optimal control

\section{INTRODUCTION}

\begin{abstract}
Ample research has been devoted to improving vehicle's operational efficiency and decreasing its environmental footprint. Suggested solutions, whose acceptance has been accelerated recently, are hybrid electric vehicles (HEVs) and plug-in HEVs [Guzzella and Sciarretta, 2013].
\end{abstract}

HEVs' powertrains utilize an energy buffer, typically a battery and/or a supercapacitor, and one or more electric machines (EMs) to supplement the output of the internal combustion engine (ICE). The multiple power sources open the possibility to further improve operational efficiency and customize vehicle powertrains. However, this also increases vehicle complexity, bringing into play many vehicle attributes, such as design, operation, performance, lifetime, and the interplay with the infrastructure, such as systems for navigation, traffic information, and the electric grid. Moreover, these attributes are strongly coupled as, for example, the way the vehicle is operated may affect the choice and size of powertrain components, and vice versa.

HEV studies where many vehicle attributes are highly intertwined are common in the early design phase of the vehicle. Typical examples are estimation of total cost of ownership, optimal sizing of powertrain components, case studies over pricing scenarios, etc. A common bottleneck that these studies encounter, in terms of computational time, is optimization of the energy management. The energy management decides, among other things, the optimal power split between the ICE and the EMs that minimizes a certain performance index. A typical performance index is fuel consumption that has to be minimized over a certain driving cycle, or a set of such cycles.

In order to assess HEV studies that are not biased in terms of HEV operation, the energy management problem is typically re-optimized many times, for all studied driving cycles, charging infrastructures, performance requirements, pricing scenarios, and sizes and types of powertrain components [Kim and Peng, 2007, Moura et al., 2010, Murgovski et al., 2012c, Ebbesen et al., 2012, Pourabdollah et al., 2013, Hu et al., 2014]. However, this inevitably leads to a computational overload, if for example, energy management is optimized with an algorithm that guarantees global optimality, such as dynamic programming (DP) [Bellman, 1957].

For the purpose of relaxing the computational burden, researchers have sought sub-optimal control strategies. In this category belong rule based and heuristic strategies [Fellini et al., 1999, Galdi et al., 2001, Wu et al., 2011]. The well known equivalent consumption minimization strategy [Guzzella and Sciarretta, 2013] can also be seen as a suboptimal control strategy, besides its clear efficacy in realtime control.

An alternative sub-optimal approach has been presented by Murgovski et al. [2012c, 2014]. The strategy relies on approximating component losses as convex in power and energy, which enables convex optimization to be used to simultaneously size powertrain components and optimize energy management. Integer decisions, such as engine on/off and gear selection, are decided by heuristics; initial steps have been taken towards a more elaborate control that may lead to a near optimal solution [Murgovski et al., 2013, Elbert et al., 2014]. 


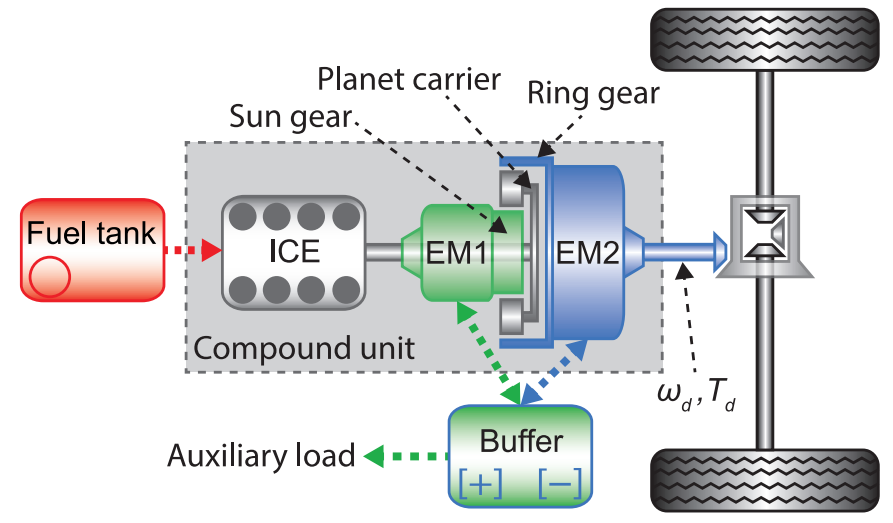

Fig. 1. Series-parallel HEV powertrain model.

This paper extends the work of Murgovski et al. [2012c, 2014], by proposing modeling steps that allow convex optimization to be applied for obtaining the optimal power split of an HEV with a planetary gear set. The energy management problem is first reformulated to become separable in space (optimization variables). Then, the problem is decomposed into two optimization problems. The first is a static problem that looks for the optimal engine speed that maximizes efficiency of a compound unit, resembling an engine-generator unit combining the planetary gear and kinetic energy converters connected to it. The second is a dynamic optimization problem deciding the optimal power split between an electric buffer and the compound unit. By approximating the losses of the compound unit as convex, second order polynomial in generated power, we are able to solve the power split problem in less than 2 seconds, when the engine on/off sequence is known in advance. By comparing results with DP, we observed an approximation error of less than $0.2 \%$.

The paper is outlined as follows: problem formulation and modeling details are described in Section 2; the convex modeling steps are discussed in Section 3; an optimization example is given in Section 4; and the paper is ended with discussion and conclusion in Section 5.

\section{MODELING AND PROBLEM FORMULATION}

The vehicle powertrain is of a series-parallel topology in which the torque coupling device is a planetary gear, as illustrated in Fig. 1. The powertrain consists of an electric buffer and three kinetic energy converters, including an ICE connected to the planets carrier, and two EMs, one attached to the sun gear, EM1, and the other to the ring gear, EM2.

The vehicle is required to exactly follow a driving cycle fully described by road altitude and demanded velocity at each point in time. In the view of the vehicle powertrain this can be translated to demanded speed $\omega_{d}=\omega_{2}$ and torque $T_{d}$ on the shaft between the differential gear and EM2. Likewise, we use the symbols $\omega$ and $T$ to denote speed and torque of the three kinetic energy converters mentioned above, while $P$ and $B$ denote power, and power losses, respectively. The subscripts $E, 1,2$, and $B$, next to these signals, identify the component they describe, that is, ICE, EM1, EM2 and electric buffer, respectively. These
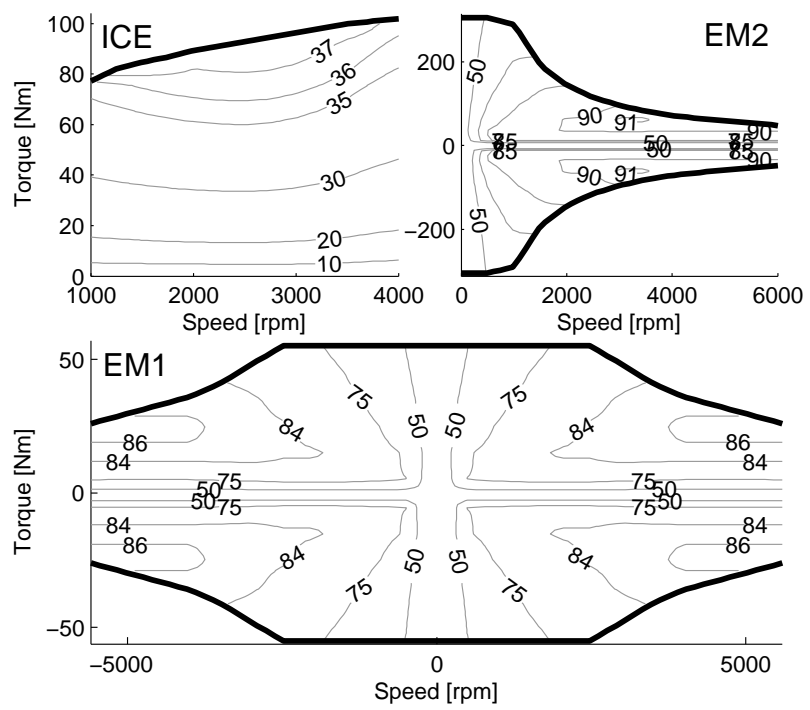

Fig. 2. Kinetic energy converters. The contour lines show efficiency in [\%]. The thick lines are torque limits.

are time dependent signals, but we omit explicit notation, in favor of increasing readability of equations that follow.

The losses of the kinetic energy converters are described by static maps, $B_{E}\left(\omega_{E}, T_{E}\right), B_{1}\left(\omega_{1}, T_{1}\right)$ and $B_{2}\left(\omega_{2}, T_{2}\right)$. Their speeds and torques are bounded by

$$
\begin{aligned}
& \omega_{E} \in\left[\omega_{\text {idle }}, \omega_{\text {Emax }}\right] \\
& \omega_{1} \in\left[-\omega_{1 \max }, \omega_{1 \max }\right] \\
& \omega_{2} \in\left[0, \omega_{2 \max }\right] \\
& T_{E} \in\left[0, T_{E \max }\left(\omega_{E}\right)\right] \\
& T_{1} \in\left[T_{1 \min }\left(\omega_{1}\right), T_{1 \max }\left(\omega_{1}\right)\right] \\
& T_{2} \in\left[T_{2 \min }\left(\omega_{2}\right), T_{2 \max }\left(\omega_{2}\right)\right]
\end{aligned}
$$

with speed dependent torque limits (also given as static maps, see Fig. 2). The engine idling speed is denoted by $\omega_{\text {idle }}$.

When formulating the optimization problem below, we consider only the case when the ICE is never turned off along the driving mission. The possibility of turning the ICE off is considered later, in Section 3.3. Furthermore, we have neglected the rotational inertia of the kinetic energy converters, since the dominating inertia is the vehicle itself. Losses of the planetary gear are also neglected.

The electric buffer, which is considered here as either a battery or a supercapacitor, is described by a total of $n$ identical cells (or modules), each represented by a voltage source and constant internal resistance $R$. In the case of a battery, it is assumed that the open circuit voltage is affine in state of charge (SOC). This is a valid assumption, as long as the battery is not operated at very low and high SOC, which is typically the case with HEVs [Guzzella and Sciarretta, 2013, Murgovski et al., 2012b]. Then, the buffer energy described in terms of the cell's terminal voltage $u$ is

$$
E_{B}=n \frac{C}{2}\left(u^{2}-u_{0}^{2}\right)
$$

where $C$ is cell's capacity in $[\mathrm{F}]$, and $u_{0}$ is the battery cell open circuit voltage at the minimum operating SOC. By deriving the terminal voltage above as a function of energy, the buffer losses and power limits can be formulated as 


$$
\begin{aligned}
& B_{B}\left(E_{B}, P_{B}\right)=R C \frac{P_{B}^{2}}{2 E_{B}+C u_{0}^{2} n} \\
& P_{B} \in\left[i_{\text {min }}, i_{\text {max }}\right] \sqrt{n\left(\frac{2}{C} E_{B}+u_{0}^{2} n\right)}
\end{aligned}
$$

where $i_{\min }$ and $i_{\max }$ are maximum charging and discharging current of a buffer cell, and $P_{B}$ is internal power of the pack. By letting $u_{0}=0$, these equations describe a supercapacitor, while by letting $C \rightarrow \infty$ they describe a battery with a constant open circuit voltage. As the dominator in (7) is positive, the losses are convex in $P_{B}, E_{B}$ and $n$ (although in this study $n$ is considered a constant, rather than an optimization variable). Similarly, the square root function in (8) is concave in $E_{B}$ and $n$. More detailed derivation of the buffer model can be found in [Murgovski et al., 2012b, Egardt et al., 2014].

The optimization objective is minimization of fuel consumption, where fuel power is computed as

$$
P_{f}\left(\omega_{E}, T_{E}\right)=\omega_{E} T_{E}+B_{E}\left(\omega_{E}, T_{E}\right) .
$$

Finally, the optimization problem can be formulated as

$$
\begin{aligned}
& \min _{\omega_{E}, T_{E}, P_{B}} \int_{0}^{t_{f}} P_{f}\left(\omega_{E}, T_{E}\right) d t \\
& \text { subject to: }(1-6,8), \\
& \omega_{1}=(1+r) \omega_{E}-r \omega_{2} \\
& T_{E}=-(1+r) T_{1} \\
& \omega_{E} T_{E}+\omega_{1} T_{1}+\omega_{2} T_{2}=\omega_{2}\left(T_{d}+T_{b r k}\right) \\
& P_{B}=\omega_{1} T_{1}+\omega_{2} T_{2}+B_{1}\left(\omega_{1}, T_{1}\right) \\
& \quad+B_{2}\left(\omega_{2}, T_{2}\right)+B_{B}\left(E_{B}, P_{B}\right)+P_{a} \\
& \dot{E}_{B}=-P_{B} \\
& E_{B} \in n\left[E_{B \min }, E_{B \max }\right] \\
& E_{B}(0)=E_{B}\left(t_{f}\right)=E_{B 0} \\
& T_{b r k} \geq 0 .
\end{aligned}
$$

The constraints (10a) and (10b) arise from the speed and torque coupling of the planetary gear, where $r$ is a scalar denoting the ratio of the number of teeth of the ring gear, vs. number of teeth of the sun gear. The constraints (10c) and (10d) represent mechanical and electrical power balance, where $T_{b r k}$ is torque applied to the friction brakes, and $P_{a}$ is power consumed by auxiliary devices. The buffer energy limits are described by (10f), and (10g) is imposed to sustain the initial battery energy $E_{B 0}$ at the end of the driving cycle. The time the vehicle finishes the driving mission is denoted by $t_{f}$. We have chosen a minimal set of optimization variables, $\omega_{E}, T_{E}$ and $P_{B}$, considering that all other signals can be derived from the equality constraints. The time dependent signals $\omega_{2}, T_{d}, P_{a}$, and the scalars $r, n$, are pre-decided.

\section{CONVEX MODELING}

The problem (10) is a non-convex dynamic optimization problem. Traditionally, this problem is solved by DP, but in order to decrease computational time we seek a solution based on convex optimization. A common convexifying step that fits well original data is approximating the losses of the kinetic energy converters as convex second order polynomials in torque, with coefficients parameterized in speed, [Murgovski et al., 2012c, Guzzella and Sciarretta,
2013]. Although this step has been successfully applied to series and parallel powertrains [Murgovski et al., 2012c], it is clear that it is not appropriate here, because in (10) engine speed is not a known signal, but an optimization variable that is yet to be determined. Hence, the first step towards convexification, described below, attempts decoupling $\omega_{E}$ from the dynamic problem (10).

\subsection{Separation in space}

The signals $\omega_{1}, T_{1}$ and $T_{2}$ can be removed from (10) by deriving them from (10a-10c) as functions of $\omega_{E}, T_{E}, \omega_{2}$, $T_{d}$ and $T_{b r k}$. The speed and torque limits of the kinetic energy converters (1-6) will now translate to constraints on $\omega_{E}$ and $T_{E}$. We present these constraints here, for didactic reasons, although it will become clear to the end of this section that they will not be explicitly needed in the final problem reformulation.

The ICE speed and torque limits are now

$$
\begin{aligned}
\omega_{E} & \geq \max \left\{\omega_{\text {idle }}, \frac{r \omega_{2}-\omega_{1 \max }}{1+r}\right\} \\
\omega_{E} \leq \min & \left\{\omega_{\text {Emax }}, \frac{r \omega_{2}+\omega_{1 \max }}{1+r}\right\} \\
T_{E} \geq \max & \left\{0, \frac{1+r}{r}\left(T_{d}-T_{2 \max }\left(\omega_{2}\right)\right)\right\} \\
T_{E} \leq \min & \left\{T_{E \max }\left(\omega_{E}\right), \frac{1+r}{r}\left(T_{d}-T_{2 \min }\left(\omega_{2}\right)\right),\right. \\
& \left.-(1+r) T_{1 \min }\left((1+r) \omega_{E}-r \omega_{2}\right)\right\} .
\end{aligned}
$$

The braking torque $T_{b r k}$ does not make a qualitative difference in (12) and it has therefore been removed. These constraints give a clearer picture of how the ICE is operated. For example, (11) reveals that at high vehicle speeds the ICE may have to be rotated with greater than idling speed, even when no engine torque is required. From (12) it is clear that the ICE has to deliver torque when EM2 cannot satisfy the demands alone.

The power balance (10d) will also change, taking the form

$$
\begin{aligned}
P_{B} & =\omega_{2}\left(T_{d}+T_{b r k}\right)-\omega_{E} T_{E}+B_{1}\left(\omega_{2}, \omega_{E}, T_{E}\right) \\
& +B_{2}\left(\omega_{2}, T_{E}, T_{d}, T_{b r k}\right)+B_{B}\left(E_{B}, P_{B}\right)+P_{a} .
\end{aligned}
$$

Now, the same trick proposed by Murgovski et al. [2012c] can be used, to relax (13) with inequality. This will enable dropping off $T_{b r k}$ from (13) without any loss of generality. Namely, we allow the buffer to deliver more power than needed by the right side of (13). At the optimum, this constraint will hold with equality at each time instance where $T_{d}$ is positive. In fact, the constraint will hold with inequality only when $T_{d}$ is negative, and not all braking energy can be recuperated because either the EM1 and EM2 torque limits are active, or the battery charges with maximum allowed current. More rigorous proof can be found in [Egardt et al., 2014].

The energy management problem can now be summarized as

$$
\begin{aligned}
& \min _{\omega_{E}, T_{E}, P_{B}} \int_{0}^{t_{f}} P_{f}\left(\omega_{E}, T_{E}\right) d t \\
& \text { subject to: }(8,10 \mathrm{e}, 10 \mathrm{f}, 10 \mathrm{~g}, 11,12), \\
& P_{B} \geq \omega_{2} T_{d}-\omega_{E} T_{E}+B_{1}\left(\omega_{2}, \omega_{E}, T_{E}\right) \\
& \quad+B_{2}\left(\omega_{2}, T_{E}, T_{d}\right)+B_{B}\left(E_{B}, P_{B}\right)+P_{a}
\end{aligned}
$$


Next, we introduce a variable change

$$
P_{C}=\omega_{E} T_{E}-B_{1}\left(\omega_{2}, \omega_{E}, T_{E}\right)-B_{2}\left(\omega_{2}, T_{E}, T_{d}\right)
$$

that replaces $T_{E}$. The fuel power becomes a function of four arguments, $P_{f}\left(\omega_{2}, \omega_{E}, T_{d}, P_{C}\right)$, while the ICE torque limits (12) transform to

$$
P_{C} \in\left[P_{C \min }\left(\omega_{2}, \omega_{E}, T_{d}\right), P_{C \max }\left(\omega_{2}, \omega_{E}, T_{d}\right)\right] .
$$

Since the power losses $B_{1}(\cdot), B_{2}(\cdot)$ and $B_{E}(\cdot)$ are given as static maps, the functions $P_{f}(\cdot), P_{C \min }(\cdot), P_{C \max }(\cdot)$ are also static maps. The important point here is to realize that the fuel power can be written as

$$
\begin{aligned}
& P_{f}(\cdot)=P_{C}+B_{C}(\cdot) \\
& B_{C}(\cdot)=B_{E}(\cdot)+B_{1}(\cdot)+B_{2}(\cdot)
\end{aligned}
$$

resembling an energy converter that is a compound unit including the ICE, EM1 and EM2 (hence, includes their losses), whose power is limited both by the speed/torque limits of the energy converters, and the speed/torque driving demands. It becomes also evident that the engine speed $\omega_{E}$ resides only in the objective function and in the boxing constraints $(11,15)$. Thus, it is possible to reformulate the optimization problem as a bilevel program

$$
\begin{aligned}
& \min _{P_{C}, P_{B}} \int_{0}^{t_{f}} P_{f}\left(\omega_{2}, \omega_{E}^{*}, T_{d}, P_{C}\right) d t \\
& \text { subject to: }(8,10 \mathrm{e}, 10 \mathrm{f}, 10 \mathrm{~g}), \\
& P_{B}+P_{C} \geq \omega_{2} T_{d}+B_{B}\left(E_{B}, P_{B}\right)+P_{a} \\
& P_{C} \in\left[P_{C \min }\left(\omega_{2}, \omega_{E}^{*}, T_{d}\right), P_{C \max }\left(\omega_{2}, \omega_{E}^{*}, T_{d}\right)\right] \\
& \omega_{E}^{*}=\underset{\omega_{E}}{\operatorname{argmin}} P_{f}\left(\omega_{2}, \omega_{E}, T_{d}, P_{C}\right) \\
& \quad \text { subject to: }(11), \\
& \quad P_{C} \in\left[P_{C \min }\left(\omega_{2}, \omega_{E}, T_{d}\right), P_{C \max }\left(\omega_{2}, \omega_{E}, T_{d}\right)\right] .
\end{aligned}
$$

The lower-level task is a static optimization problem that can be separated from the upper-level dynamic optimization problem. Its optimal solution $\omega_{E}^{*}$ is not a value, but rather a function of $\omega_{2}, T_{d}, P_{C}$. Given that the energy converter losses are static maps, the lower-level optimization task can be solved by first gridding the feasible sets for $\omega_{2}, T_{d}, P_{C}$, and then obtaining $\omega_{E}^{*}$ for each gridded combination. This is an exhaustive search procedure, but it can be performed independently of the upper-level task. The lower-level task can be formulated as

$$
\omega_{E}^{*}\left(\omega_{2}, T_{d}, P_{C}\right)=\underset{\omega_{E}}{\operatorname{argmin}} P_{f}\left(\omega_{2}, \omega_{E}, T_{d}, P_{C}\right)
$$

subject to:

$$
\begin{aligned}
\omega_{E} \in \mathcal{W}_{E} \subseteq & {\left[\max \left\{\omega_{\text {idle }}, \frac{r \omega_{2}-\omega_{1 \max }}{1+r}\right\},\right.} \\
& \left.\min \left\{\omega_{\text {Emax }}, \frac{r \omega_{2}+\omega_{1 \max }}{1+r}\right\}\right]
\end{aligned}
$$

$$
\begin{aligned}
& P_{C} \in \mathcal{P}_{C}\left(\omega_{2}, \omega_{E}, T_{d}\right) \\
& \omega_{2} \in \mathcal{W}_{2} \subseteq\left[0, \omega_{2 \max }\right] \\
& T_{d} \in \mathcal{T}_{d} .
\end{aligned}
$$

where the sets $\mathcal{W}_{E}, \mathcal{P}_{C}, \mathcal{W}_{2}, \mathcal{T}_{d}$ are discrete. This problem is solved numerically; further detailed in Appendix A.

As a consequence, one dimension can be removed from the fuel power function of the upper-level task

$$
\tilde{P}_{f}\left(\omega_{2}, T_{d}, P_{C}\right)=P_{f}\left(\omega_{2}, \omega_{E}^{*}\left(\omega_{2}, T_{d}, P_{C}\right), T_{d}, P_{C}\right),
$$

reflecting the compound unit operated at the optimal speed $\omega_{E}$ for any $\omega_{2}, T_{d}, P_{C}$. The power bounds $P_{C \min }(\cdot)$,

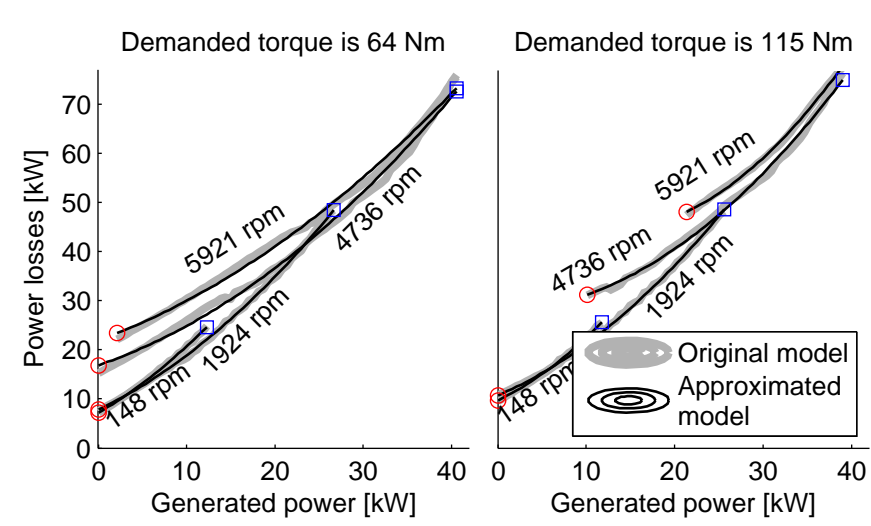

Fig. 3. Original and approximated power losses of the compound unit, plotted for two demanded torques (subplots), and four demanded speeds (contour lines). The minimum and maximum generated power are depicted by circle and square, respectively.

$P_{C \max }(\cdot)$ are affected similarly, resulting in the following dynamic optimization problem

$$
\begin{aligned}
& \min _{P_{C}, P_{B}} \int_{0}^{t_{f}} \tilde{P}_{f}\left(\omega_{2}, T_{d}, P_{C}\right) d t \\
& \text { subject to: }(8,10 \mathrm{e}, 10 \mathrm{f}, 10 \mathrm{~g}), \\
& P_{B}+P_{C} \geq \omega_{2} T_{d}+B_{B}\left(E_{B}, P_{B}\right)+P_{a} \\
& P_{C} \in\left[\tilde{P}_{C \min }\left(\omega_{2}, T_{d}\right), \tilde{P}_{C \max }\left(\omega_{2}, T_{d}\right)\right] .
\end{aligned}
$$

The optimization problem (19) shows some similarities to the energy management problem for an $\mathrm{HEV}$ in a series powertrain topology. The difference is that the compound unit, which resembles an engine-generator unit in a series powertrain, is here parameterized in $\omega_{2}$ and $T_{d}$.

\subsection{Approximation of power losses}

Power losses of the compound unit, constructed from the kinetic energy converters in Fig. 2, are depicted in Fig. 3. The figure illustrates losses for two different demanded torques and four demanded speeds. It can be observed that the losses appear convex in generated power. In fact, the figure also illustrates losses of an approximated model, quadratic in power, which fit well the original losses. Thus, the fuel power of the compound unit can be expressed as

$$
\tilde{P}_{f}(\cdot) \approx a_{0}\left(\omega_{2}, T_{d}\right)+a_{1}\left(\omega_{2}, T_{d}\right) P_{C}+a_{2}\left(\omega_{2}, T_{d}\right) P_{C}^{2}
$$

with coefficients parameterized in $\omega_{2}, T_{d}$. For speed/torque inputs that are not grid nodes in $\mathcal{W}_{2}, \mathcal{T}_{d}$, the values of these coefficients are obtained by linear interpolation. Finally, replacing (20) in (19) gives a convex second order cone program that can be solved efficiently [Murgovski, 2012].

Obtaining the convex model (20) is not surprising. In fact, similar procedure, and similar outcome, has been encountered previously, when approximating losses of enginegenerator units in series HEV powertrains [Murgovski et al., 2012c].

\subsection{Engine on/off control}

A limitation of the convex power-split problem (19) is that it cannot handle engine on/off control. Although more elaborate procedures for solving this problem have 
already been published, [Murgovski et al., 2013, Elbert et al., 2014], we propose here a simple heuristic decision that tends to give near optimal results. First, we present remaining modeling details for the scenario where ICE is turned off.

Since the HEV powertrain presented in Fig. 1 is devoid of clutch, the ICE cannot be mechanically decoupled from the wheels. Hence, in cases where ICE is off and rotating, it becomes a load to the system due to its friction losses. We modeled this by a constant friction torque. Furthermore, as the ICE friction losses are greater than the friction losses of EM1, it is favorable to keep ICE still, and rotate EM1 instead. This can be achieved as long as EM1 is not overspeeded. Then, ICE's speed is computed as

$$
\omega_{E}=\max \left\{0, \frac{r \omega_{2}-\omega_{1 \max }}{1+r}\right\} .
$$

However, when this speed exceeds a limit $\omega_{E} \geq \omega_{\text {Eon }}$, the ICE friction losses become greater than its idling losses. Then, it is beneficial to turn the ICE on, and let (19) decide its power.

The speed limit $\omega_{E o n}$ can be translated to a limit on demanded speed

$$
\omega_{o n}=\frac{(1+r) \omega_{E o n}+\omega_{1 \max }}{r} .
$$

This is the final ingredient for the ICE on/off heuristics, which we formulate as follows

$$
\text { ICE is } \begin{cases}\text { on, } & \omega_{2} T_{d} \geq P_{\text {on }} \text { or } \omega_{2} \geq \omega_{\text {on }}, \\ \text { off, } & \text { otherwise. }\end{cases}
$$

The speed threshold $\omega_{\text {on }}$ is easy to obtain from the static ICE model. The power threshold $P_{\text {on }}$ is obtained by solving the convex problem (19) with on/off sequences generated using several power threshold. Then, the power threshold is chosen that minimizes fuel consumption for the studied driving cycle.

At time instances where ICE is off, the optimization variable $P_{C}$ is removed from the problem (19), and the only remaining variable is $P_{B}$.

\section{OPTIMIZATION EXAMPLE}

We apply the proposed optimization method to obtaining the optimal energy management of a Toyota Prius hybrid, driven on the New European Driving Cycle. The kinetic energy converters are as described in Fig. 2, while the remaining vehicle data has been obtained from ADVISOR [2003]. The electric buffer is a battery with SOC confined within $25-75 \%$, and initial and final charge of $50 \%$.

Three different scenarios are considered: first the ICE is never turned off; second, we allow the ICE to be turned off, but the on/off sequence is decided by the heuristics presented in Section 3.3; and third, we obtain the optimal ICE on/off control. The energy management problem in the last scenario is solved by DP, while for the remaining two scenarios the problem is solved by both convex optimization and DP. The optimal fuel consumption is given in Table 1, along with difference in fuel consumption between convex optimization and DP. For the third scenario in Table 1, where convex optimization cannot deliver the optimal on/off control, fuel consumption is obtained by the on/off heuristics.
Table 1. Optimal fuel consumption obtained by convex optimization and DP.

\begin{tabular}{llll}
\hline ICE on/off & Convex & DP & Difference \\
scenario & {$[1 / 100 \mathrm{~km}]$} & {$[1 / 100 \mathrm{~km}]$} & {$[\%]$} \\
ICE is always on & 4.49 & 4.48 & 0.16 \\
Heuristic on/off & 3.31 & 3.30 & 0.10 \\
Optimal on/off & 3.31 & 3.28 & 0.89 \\
\hline
\end{tabular}

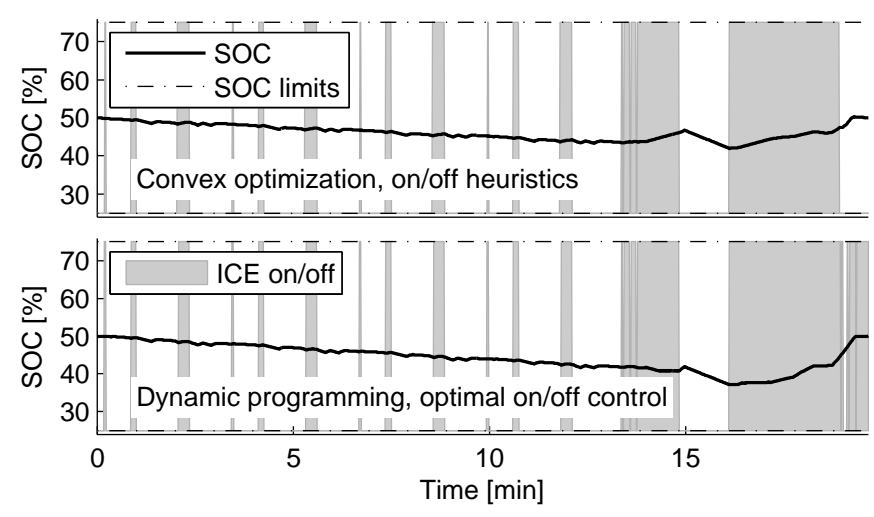

Fig. 4. Battery SOC and engine on/off obtained by convex optimization and DP.

The results indicate that the error due to approximation of the power losses (20) is very small, in the order of $0.15 \%$. The on/off heuristics give more visible error. (The difference in on/off signals is also depicted in Fig. 4.) However, the error is reasonably small, below $1 \%$.

The average computational time DP needs to solve the energy management problem is about $50 \mathrm{~min}$, on a standard PC (4 GB RAM, 2.67 GHz dual core CPU). (The optimization time could be shortened if dedicated solver is used.) The optimization problem in DP has been formulated with two control signals $\omega_{E}$ and $T_{E}$, and one state, the battery SOC; all three having their feasible range gridded with 200 points. The sampling time used both in DP and convex optimization is $1 \mathrm{~s}$.

The computational time convex optimization needs to solve the power split problem is typically less than $2 \mathrm{~s}$ $(1.8 \mathrm{~s}$ in average). This problem is solved several times to obtain the optimal power threshold required by the ICE on/off heuristics. (Using a resolution of $1 \mathrm{~kW}$ it is found that the optimal power threshold is $3 \mathrm{~kW}$, while $\omega_{\text {on }} \approx 3540 \mathrm{rpm}$.) However, the convex optimization also requires the static map $\tilde{P}_{f}(\cdot)$ of the compound unit. This map has to be generated once, and does not need to be changed for different case studies (as long as the kinetic energy converters and planetary gear are not changed). Obtaining the map needs about $30 \mathrm{~min}$, when 100 grid points are used for $\mathcal{W}_{E}$ and $\mathcal{P}_{C}$ and 60 grid points for $\mathcal{W}_{2}$ and $\mathcal{T}_{d}$. The approximation of $\tilde{P}_{f}(\cdot)$ is a simple least-square problem. With constraints included to ensure convexity and positive idling losses, the time needed to approximate $\tilde{P}_{f}(\cdot)$ is $3 \mathrm{~s}$.

Note that $\tilde{P}_{f}(\cdot)$ can be generated in significantly shorter time if the HEV is to be simulated only on one driving cycle. Then, the compound unit can be created only for speed/torque combinations that describe the driving cycle. 


\section{DISCUSSION AND CONCLUSION}

We proposed a method for computationally efficient energy management of series-parallel HEVs with a planetary gear. By decoupling the energy management problem, our study showed that it is possible to shift the computational effort to an off-line generation of a compound unit, leaving a convex dynamic power split problem that can be solved in less than $2 \mathrm{~s}$. This makes it possible to perform HEV assessment studies, which may otherwise require impractically high computational demands. Furthermore, this method could also be considered for a real-time HEV energy management (onboard the vehicle), in e.g. model predictive control fashion.

A limitation of this method can be seen when sizing the kinetic energy converters or the planetary gear, as this will require re-generation of the compound unit. It is possible, however, to scale the compound unit and the electric buffer simultaneously with the optimization of the energy management. Moreover, convexity is preserved even when more energy buffers are added to the system, e.g. a dual system consisting of battery and supercapacitor (see e.g. [Murgovski et al., 2012a] for hints on this). A detailed investigation on simultaneous optimization of components sizing and energy management will be considered in future studies.

\section{REFERENCES}

ADVISOR. Advanced vehicle simulator. http://bigladdersoftware.com/advisor, 2003.

R. Bellman. Dynamic Programming. Princeton Univ Pr, New Jersey, June 1957.

S. Ebbesen, C. Dönitz, and L. Guzzella. Particle swarm optimisation for hybrid electric drive-train sizing. Int. J. Vehicle Design, 58(58):181-199, 2012.

B. Egardt, N. Murgovski, M. Pourabdollah, and L. Johannesson. Electromobility studies based on convex optimization: Design and control issues regarding vehicle electrification. IEEE Control Systems Magazine, 34(2): 32-49, 2014.

P. Elbert, T. Nüesch, A. Ritter, N. Murgovski, and L. Guzzella. Engine on/off control for the energy management of a serial hybrid electric bus via convex optimization. IEEE Transactions on Vehicular Technology, 2014. doi:10.1109/TVT.2014.2304137.

A. D. Fellini, D. Assanis, G. Delagrammatikas, R. Fellini, Z. Filipi, J. Liedtke, N. Michelena, P. Papalambros, D. Reyes, D. Rosenbaum, A. Sales, and M. Sasena. An optimization approach to hybrid electric propulsion system design. In Journal of Mechanics of Structures and Machines, Automotive Research Center Special Edition, pages 393-421, 1999.

V. Galdi, L. Ippolito, A. Piccolo, and A. Vaccaro. A genetic-based methodology for hybrid electric vehicles sizing. Soft Computing - A Fusion of Foundations, Methodologies and Applications, 5(6):451-457, 2001.

L. Guzzella and A. Sciarretta. Vehicle propulsion systems. Springer, Verlag, Berlin, Heidelberg, 3 edition, 2013.

X. Hu, N. Murgovski, L. Johannesson, and B. Egardt. Comparison of three electrochemical energy buffers applied to a hybrid bus powertrain with simultaneous optimal sizing and energy management. IEEE Transactions on Intelligent Transportation Systems, 2014. doi:10.1109/TITS.2013.2294675.
M. Kim and H. Peng. Power management and design optimization of fuel cell/battery hybrid vehicles. Journal of Power Sources, 165(2):819-832, 2007.

S. J. Moura, D. S. Callaway, H. K. Fathy, and J. L. Stein. Tradeoffs between battery energy capacity and stochastic optimal power management in plug-in hybrid electric vehicles. Journal of Power Sources, 195(9):29792988, 2010

N. Murgovski. Optimal Powertrain Dimensioning and Potential Assessment of Hybrid Electric Vehicles. PhD thesis, Chalmers University of Technology, Gothenburg, Sweden, 2012.

N. Murgovski, L. Johannesson, A. Grauers, and J. Sjöberg. Dimensioning and control of a thermally constrained double buffer plug-in HEV powertrain. In 51st IEEE Conference on Decision and Control, Maui, Hawaii, December 10-13 2012a.

N. Murgovski, L. Johannesson, and J. Sjöberg. Convex modeling of energy buffers in power control applications. In IFAC Workshop on Engine and Powertrain Control, Simulation and Modeling (E-CoSM), Rueil-Malmaison, Paris, France, October 23-25 2012b.

N. Murgovski, L. Johannesson, J. Sjöberg, and B. Egardt. Component sizing of a plug-in hybrid electric powertrain via convex optimization. Mechatronics, 22(1):106-120, 2012c.

N. Murgovski, L. Johannesson, and J. Sjöberg. Engine on/off control for dimensioning hybrid electric powertrains via convex optimization. IEEE Transactions on Vehicular Technology, 62(7):2949-2962, 2013.

N. Murgovski, L. Johannesson, and B. Egardt. Optimal battery dimensioning and control of a CVT PHEV powertrain. IEEE Transactions on Vehicular Technology, 2014. doi:10.1109/TVT.2013.2290601.

M. Pourabdollah, N. Murgovski, A. Grauers, and B. Egardt. Optimal sizing of a parallel PHEV powertrain. IEEE Transactions on Vehicular Technology, 62 (6):2469-2480, 2013.

L. Wu, Y. Wang, X. Yuan, and Z. Chen. Multiobjective optimization of HEV fuel economy and emissions using the self-adaptive differential evolution algorithm. IEEE Transactions on Vehicular Technology, 60(6):2458-2470, 2011.

\section{Appendix A. GENERATION OF COMPOUND UNIT}

The compound unit has been generated in six steps:

(1) Gridded sets $\mathcal{P}_{C}, \mathcal{W}_{2}, \mathcal{T}_{d}, \mathcal{W}_{E}$ and $\mathcal{T}_{E}$ are created. Here, $\mathcal{T}_{E}$ is a set of ICE operating torques.

(2) For each grid point in $\mathcal{W}_{2}, \mathcal{T}_{d}, \mathcal{W}_{E}, \mathcal{T}_{E}$, and based on the equalities $(10 \mathrm{a}, 10 \mathrm{~b}, 10 \mathrm{c})$ the sets $\mathcal{W}_{1}, \mathcal{T}_{1}, \mathcal{T}_{2}$ are obtained, holding operating values for $\omega_{1}, T_{1}$ and $T_{2}$.

(3) The sets $\mathcal{P}_{C s}, \mathcal{P}_{f s}$ are obtained according to $(14,16)$. These sets contain scattered data.

(4) The map $\mathcal{P}_{f}$ is obtained by Delaunay triangulation in $\mathcal{P}_{C s}, \mathcal{P}_{f s}$, for grid points in $\mathcal{P}_{C}$.

(5) Infinity is assigned to all grid points in $\mathcal{P}_{f}$ that violate any of the constraints (1-6). At the same time the minimum and maximum powers $P_{C \min }(\cdot), P_{C \max }(\cdot)$ are recorded.

(6) Finally, the map $\mathcal{P}_{f}$ is minimized with respect to $\omega_{E}$, according (17), and the three-dimensional map $\tilde{P}_{f}\left(\omega_{2}, T_{d}, P_{C}\right)$ is obtained. 